\title{
Review of brachistochrone problem as the mother of optimal control application strategy
}

\author{
S.K. Jha \\ Division of Instrumentation and Control Engineering, \\ Netaji Subhas Institute of Technology, New Delhi, India \\ Email: jhask271@gmail.com
}

Optimal control being the most important control mechanism among modern state space based control, would have been under the veil had it not been for the genesis of Brachistochrone problem by Bernoulli. Brachistochrone problem, first envisaged by Bernoulli, led to the evolution of calculus of variational approach which later on became panacea for the solution of optimal control solution strategy [1-3]. This paper discusses the evocative anecdotal details of the evolution of optimal control from brachistochrone problem. Some examples illustrate the efficacy of optimal control strategy over other classical control and other control strategy. Implication of some optimal control parameter is highlighted to display the supremacy of the controller over other available control methodology. In classical control technique, minimization of time or optimization of fuel consumption was not a matter of primary concern whereas controller designed by optimal control strategy automatically takes into consideration all these important factors. Some examples very well highlight all these features. The MATLAB and SIMULINK based graphical results are shown in Figure 1 to 3, which are used to further validation of the proposed efficacy of optimal controller.

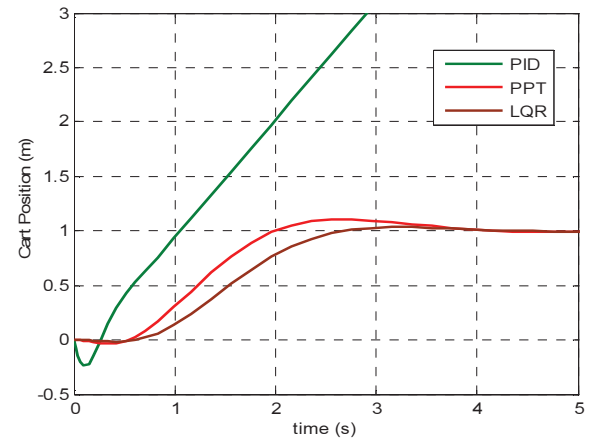

Figure 1: Response of position of cart with PID, PPT and LQR

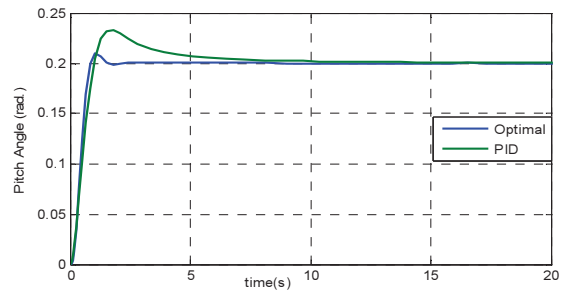

Figure 2: Pitch Angle v/s time for the PID and optimal control of aircraft

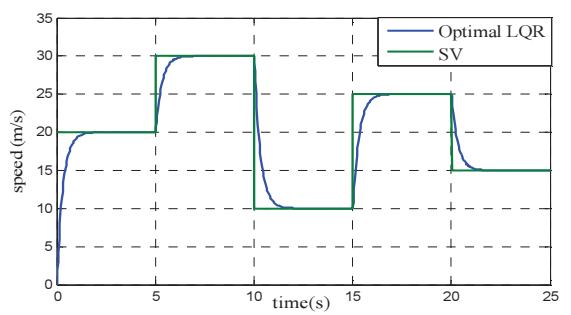

Figure 3: Optimal LQR response for vehicle

\section{References}

1. D. E. Kirk, Optimal Control Theory: An Introduction, Prentice Hall, Englewood cliffs, New Jersey, 1970.

2. S. K. Jha, H. Parthsarathy, J. R. P. Gupta and P. Gaur, "Verification of the Veracity of Brachistochrone Curve and Evolution of Optimal Control," India International Conference on Power Electronics (IICPE), N. Delhi, India, pp. 1-4, Jan. 2011.

3. S.K. Jha, A. K. Yadav, P. Gaur and J. R. P. Gupta, "LQR Based Optimal Versus Classical PID Controller for the Longitudinal Dynamics of an Aircraft," Journal of Control and Instrumentation, vol. 4, no. 2, pp. 15-23, 2013. 